\title{
Characterization of Silicone Oil used in HV Cable Sealing Ends
}

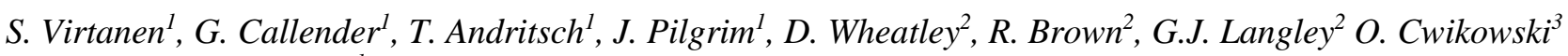 \\ ${ }^{1}$ ECS; University of Southampton, Southampton, United Kingdom. \\ ${ }^{2}$ Department of Chemistry, University of Southampton, Southampton, United Kingdom \\ ${ }^{3}$ National Grid Electricity Transmission, United Kingdom
}

\begin{abstract}
Existing polymeric cable sealing end (CSE) typically contain silicone oil as an insulating liquid between the cable core and the outside of the CSE. In recent years, a number of performance issues have been identified with silicone oil filled CSEs. To understand the reasons for the observed behavior of silicone oil, a range of characterization methods are reviewed, to allow investigation of the influence of moisture, potential chemical contaminants and the compatibility with CSE materials. The water content of all the oils studied was above the limit given in IEC 60836 (2015) for new silicone oil. There is no significant difference between the moisture content, whether the oil is used in CSE or new. Neither did the Fourier infrared (FTIR) spectroscopic analysis show difference between the oils. Preliminary results from gas chromatography connected to mass spectrometer (GC/MS) clearly show structural differences between unused expired oil and new oil samples particularly that species suspected to be cyclic siloxane appear to be present in the expired sample.
\end{abstract}

\section{Introduction}

Silicone oil is used in CSE as an insulating liquid, due to its very low dielectric losses and high resistivity. When considering dielectric properties of liquids, it seems that often the first value presented is breakdown strength as a function of field. However, it is not appropriate for evaluating the behavior of insulating material in actual applications [1]. The measure is fundamentally crude, an example of this is that the electric field is estimated without taking into consideration the conductivity of the material. In reality a more conductive material is subject to lower electric fields and as such it can appear to have higher breakdown strength than the less conductive materials. Standard test methods for assessing breakdown voltage of liquids typically employ small electrode gaps, of the order of $\leq 2.5 \mathrm{~mm}$. The electrode configuration can vary from spherical, through VDE type "mushroom" electrodes to disc electrodes. This measurement geometry is not representative of CSE conditions. When comparing different insulating liquids, they give very similar results for a given electrode arrangement in this type of testing [2]. The aged silicone oil is reported to be more prone to partial discharges, which leads to decreased resistivity [3]. But it is hard to replicate the conditions that prevail in the CSE; and in general, the results of electrical testing are very dependent on the test geometry and conditions. Often the test setup itself is more conductive than the silicone oil. For this reason, electrical testing in a way that oil is subjected to electrical stresses and then analysed by other means, is more feasible than judging it on often non reproducible electrical tests in conditions that are not even prevailing in the CSE.

The testing of silicone oil used in CSE applications in this work focuses on chemical impurities present in the silicone oil. This study does not include the detection of gaseous byproducts, even though application of dissolved gas analysis (DGA) to study the CSEs has shown to be useful [4]. This is because the storage of the oil samples might have altered the gaseous composition. The impurities present in the oil could originate from partial discharges (PD) in the oil [5]. Possible sources of them might also be cross-linked polyethylene (XLPE). For example, acetophenone can be formed as a byproduct of crosslinking process [6]. Impurities are not likely products of thermal oxidation of the oil, as the temperatures required $\left(>150{ }^{\circ} \mathrm{C}\right)$ are not present in the CSE. The depolymerization of PDMS is most likely route of degradation at lower temperatures, especially if it is catalysed with ionic or polar impurities [7]. Moisture content of the oil has been considered by some to be the cause of failure of CSE [8], but it likely that large amounts of water would be needed to induce service breakdown [9]. The purpose of this study was to determine if there is some chemical species found in the silicone oil that has been used in CSE application which could be linked to the ageing of the oil under service. This is done by comparing the used oil composition with new and old silicone oil; that have not been subjected to electrical or thermal stresses.

\section{Methods}

All the measurements were done on two different oils that where either new, expired of their use-by -date; but not used in CSE application, or ex-service oil from CSE. This gave a total of six samples which are described in Table 1. Water content of the oils was measured using Karl Fisher (KF) titration [10]. FTIR spectroscopy was used for providing a "fingerprint" of various oils and it has been previously used to detect crosslinking by-products from XLPE [6]. GC/MS was method to identify the presence of polar contaminants and additives. 


\subsection{Water content}

Measurement of $\sim 1 \mathrm{ml}$ oil samples was carried out using an Aquamax KF Coulometric titrator (GR Scientific). Water content was determined as $\mathrm{mg} / \mathrm{kg}$ using the weight and calculated as average and standard deviation of 3 measurements.

\subsection{FTIR spectroscopy}

The attenuated total reflection (ATR) sampling technique was used to study oil samples. ATR uses a property of total internal reflection resulting in an evanescent wave. A beam of infrared light is passed through the ATR crystal in such a way that it reflects at least once off the internal surface in contact with the sample. This reflection forms the evanescent wave, which extends into the sample. The penetration depth into the sample is typically between 0.5 and $2 \mu \mathrm{m}$, with the exact value being determined by the wavelength of light, the angle of incidence and the indices of refraction for the ATR crystal and the medium being probed.

\subsection{GC/MS analysis}

In gas chromatography (GC) the principle for the separation of a mixture is passing it through a medium in which the components move at different rates in gaseous state. After the components have been separated they are detected by a mass spectrometer (MS). The chemicals acetophenone and cyclic siloxane decamethyl-cyclopentacyclosiloxane (D5) were purchased from Sigma-Aldrich. Silicone oil samples were diluted in anhydrous dichloromethane in the range of $10-11 \mathrm{mg} / \mathrm{mL}$ for GC/MS analysis, because the polydispersities and molecular weights $\left(\mathrm{M}_{\mathrm{w}}\right)$ of the oils are unknown quantities. The polydispersity is a distribution of molecular mass in a given polymer sample. GC/MS conditions were a ramp from $40{ }^{\circ} \mathrm{C}$ to $320{ }^{\circ} \mathrm{C}$ by $15{ }^{\circ} \mathrm{C} / \mathrm{min}$, holding at the top temperature for 10 minutes to ensure elution of higher $\mathrm{M}_{\mathrm{w}}$ components. MS was collected at 10-520 atomic mass units (amu). The collected spectra were analysed using the Xcalibur suite of software (v. 2.0.5).

\section{Results and discussion}

\subsection{Water content}

The water content of studied oil samples is shown in Figure 1. From Figure 1 it can be seen that the variation of water content between new oils is $15 \mathrm{mg} / \mathrm{kg}$. The water content is above the limit of $50 \mathrm{mg} / \mathrm{kg}$ given in IEC 60836 (2015). There is difference between the expired oils the and new oils: the expired oils contain slightly more water, but no significant difference in water content between whether the oil is expired or new was found. The lowest water content is in the oils that have been used in service. The fact that the water content of the exservice oils is lower may be due to the different varieties of oil in the samples. Nevertheless, the analysis does suggest that there are no large increases in water content in the CSE oils samples.

\subsection{FTIR spectroscopy}

FTIR spectra of the oils are shown in Figure 2. The spectra are vertically displaced to make the comparison easier. There is no obvious difference between the new and expired oils. This
Table 1. Sample identification of studied silicone oils.

\begin{tabular}{cc}
\multicolumn{2}{c}{ Table 1. Sample identification of studied silicone oils. } \\
\hline Sample ID & State \\
\hline NA & new oil A \\
NB & new oil B \\
EC & expired, not used oil C \\
ED & expired, not used oil D \\
SE & ex-service oil E \\
SF & ex-service oil F \\
\hline
\end{tabular}

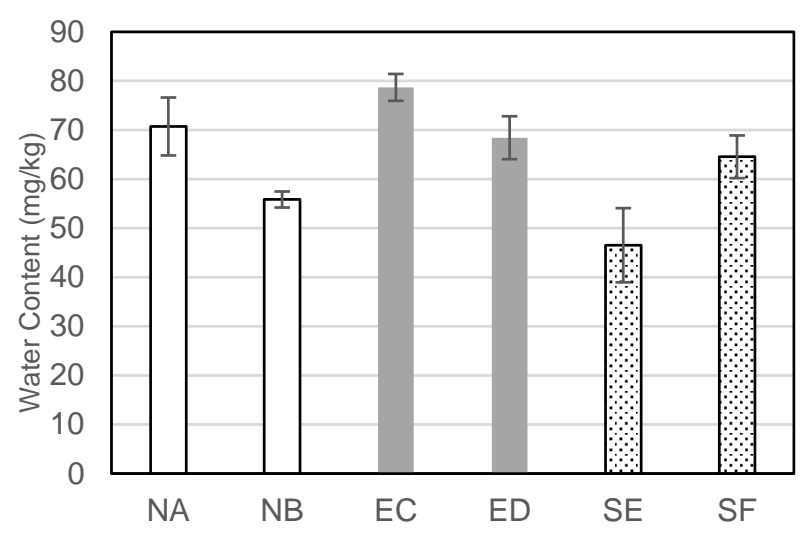

Figure 1 Water content of the studied oils.

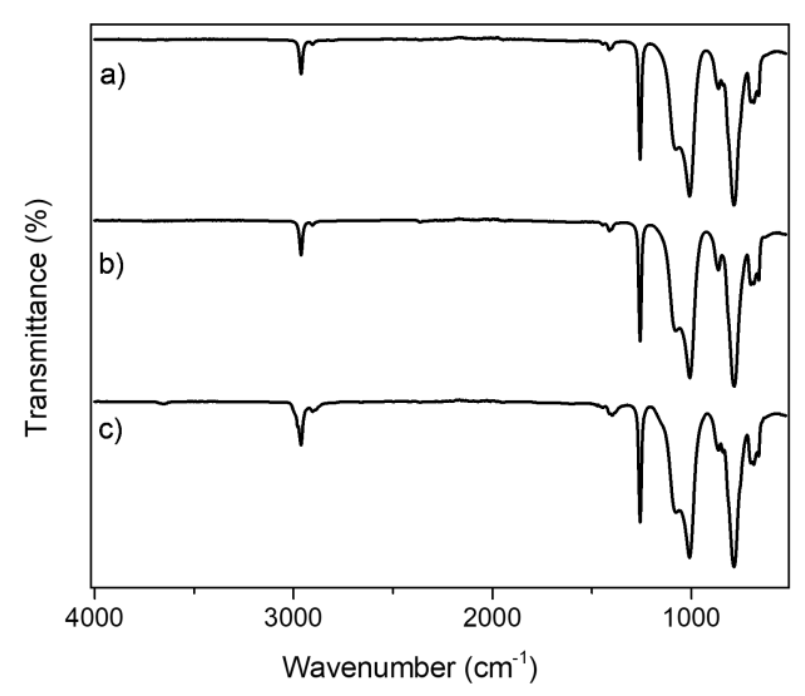

Figure 2 FTIR spectra of the studied oils: a) new oil b) expired oil c) ex-service oil. (The spectra are vertically displaced to make the comparison easier)

suggests that any by-products present due to possible ageing are so low in concentration that they cannot be detected by this method.

\subsection{GC/MS analysis}

Using individual cyclic siloxane (D5) and acetophenone it was shown that GC/MS can detect the lower molecular weight 


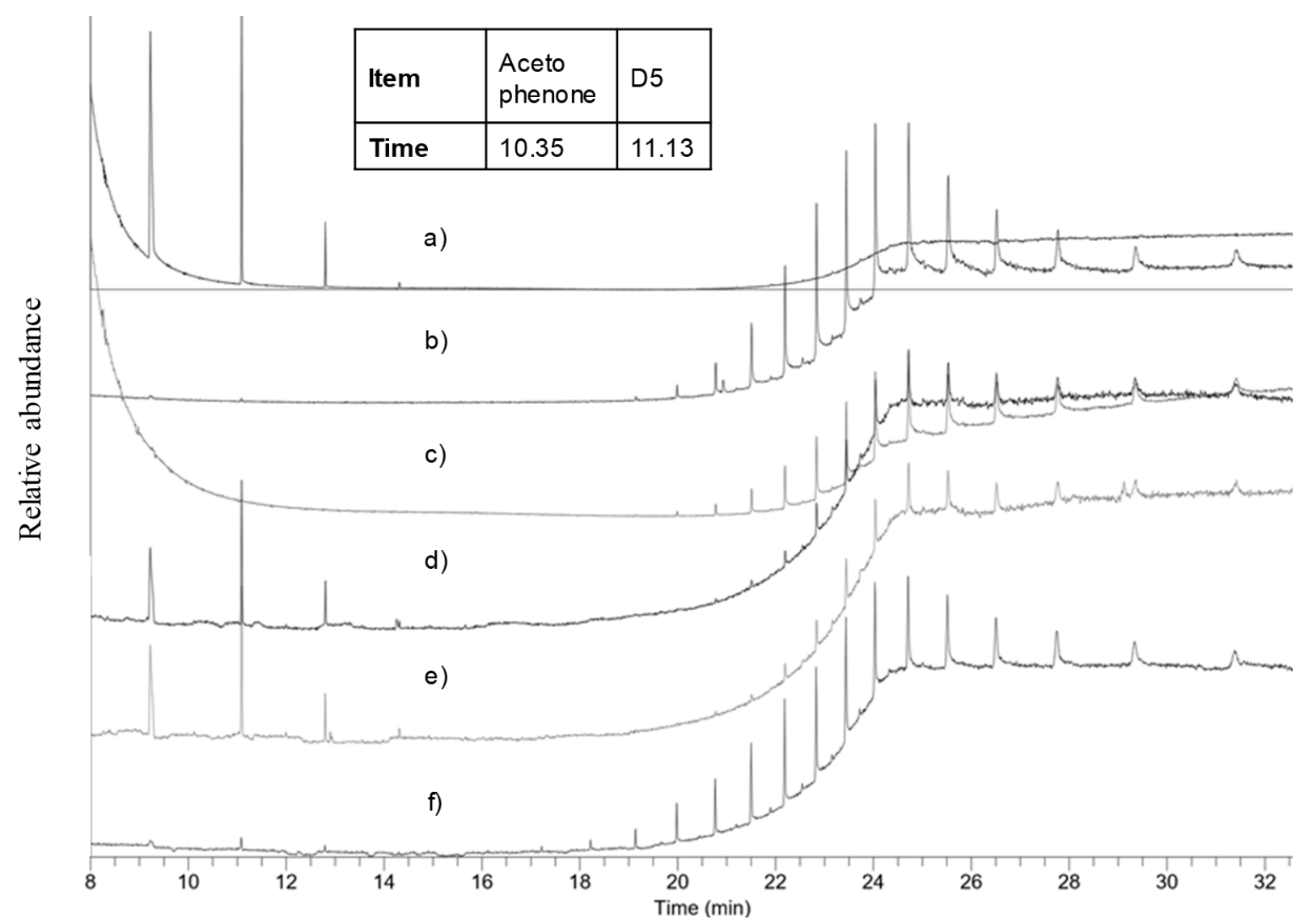

Figure 3. Retention times of possible by-products and gas chromatograms of silicone oils: a) expired EC b) new NA c) new NB d) exservice SE e) ex-service SF and f) expired ED. (offset vertically for clarity)

cyclic siloxane and XLPE crosslinking by products. The retention times used to detect possible by-products are shown in Figure 3. Figure 3 shows also the gas chromatograms of studied oils. They are vertically displaced to make the comparison easier. A distinct fingerprint pattern can be observed towards the end of the chromatogram with uniform spacing, indicative of linear siloxane oligomers. The presence of cyclic siloxane D5 is confirmed within some of the samples. The peak eluting before D5 can reasonably be assumed to be D4 by analogy, and the precession of peaks eluting after D5, are likely by extension due to be D6 and D7. The cyclic siloxane peaks at early retention times, are most abundant in expired oil EC and also seen in exservice oils; SE and SF, at varying levels of abundance. The cyclic siloxanes are products of the depolymerization of silicone oil [7]. Acetophenone is not detected in any of the oil samples. This suggests that if it is present in XLPE in contact with the silicone oil in CSE application, it has not notably transferred into the oil. Of course it is possible that the acetophenone present in oil has degraded and as a result is not detected in this analysis [6]. The lack of linear oligomer fingerprint in expired oil EC is because it is very viscous and consists of chain lengths higher than it is possible to detect in used conditions. The other expired oil is less viscous and the fingerprint of linear oligomers can be seen towards the end of the chromatogram. There is also another difference between expired oils: EC exhibits strong signal of cyclic siloxanes while for ED these peaks are hardly seen. So it is not possible to claim that cyclic siloxanes would definitely be formed during long storage time, they could possibly be present in specific oils. This emphasizes the difficulty in comparing oils with different history if they are not exactly the same oil. Both ex-service oils (SE and SF) have very similar chromatograms; containing signals from cyclic and linear siloxanes. In new oils; $\mathrm{NA}$ and NB, the signal from cyclic siloxanes is missing. The formation of cyclic oligomers seems to be related to silicone oil having been used in a CSE.

\section{Conclusions}

New, expired and silicone oil used in CSE were analysed. Spectroscopic, coulometric and chromatographic methods were used to find out any possible indication of the degradation or contamination of the oil used in CSE. There was no difference in the water content of the studied oils upon extended storage or use in CSE. The FTIR spectroscopy did not show any difference between the oils, but characteristic differences in the oils can be clearly seen by using GC/MS analysis. Preliminary GC/MS analyses show differences between different samples and the existence of cyclic siloxanes suggests that upon storage silicone oil might undergo depolymerization. When the oil is used in CSE it clearly depolymerizes, but the extent of it is still unknown. Simulation tools are currently being used to investigate in service conditions present within CSEs to inform future testing. 


\section{Acknowledgements}

The authors would like to thank the National Grid for supporting this work. All the data supporting this study are openly available from the University of Southampton repository at https://doi.org/10.5258/SOTON/D0927.

\section{References}

[1] IEC, "Standard IEC 60243-1: Electric strength of insulating materials. Test methods. Tests at power frequencies," IEC, 2013.

[2] M. Lashbrook, A. Gyore and R. Martin, "A review of the fundamental dielectric characteristics of ester-based dielectric liquids," Procedia Engineering, vol. 202, pp. 121-129, 2017.

[3] A. Haidar, J. C. Fothergill, D. L. A and P. Hopewell, "Anomalous dielectric response of very small quantities of virgin, aged and failed silicone oil,," IEEE Transactions on Dielectrics and Electrical Insulation, vol. 10 , no. 2 , pp. 336-342, 2003.

[4] N. Singh, S. Singh, R. Reyes, J. Hlavac, R. Schmidt, Uzelac, M, T. Zhao and D. Kummer, "DGA (dissolved gas analysis) diagnostic method reveals internal carbonization in oil-filled high voltage extruded cable terminations," in Jicable'15, Versailles, 2015.

[5] T. Suzuki, S. Murakami, S. Kobayashi, K. Kiyotaki and M. Umeda, "Decomposition Products of Silicone Liquid Due to Electric Discharge," IEEE Transactions on Electrical Insulation, pp. 152-157, 1983.

[6] A. Smedberg, J.-O. Boström and P. R. Wald, "Comparison of different analytical test methods to monitor crosslinking by-products in XLPE insulated cables," in 7th International Conference on Power Insulated Cables, JiCable, Versailles, 2007.

[7] P. R. Dvornic, "Thermal properties of polysiloxanes," in SiliconContaining Polymers, R.G. Jones et al. (eds.), Silicon-Containing Polymers, 185-212. (C) 2000 ., Kluwer Academic Publishers, 2000, pp. 185212 .

[8] K. Liland, S. Hvidsten, G. Birkenes, F. Mauseth, G. A. Ward and E. Bjerka, "Failure Modes and Condition Assessment of High Voltage Oil Filled XLPE Terminations," in 2009 IEEE Electrical Insulation Conference, Montreal, 2009.

[9] F. Mauseth, S. Hvidsten and G. Birkenes, "Water ingress in highvoltage cross-linked polyethylene (XLPE) cable terminations," IEEE Electrical Insulation Magazine, vol. 28, no. 5, pp. 24-31, 2012.

[10] BSI, BS EN 60814:1998, IEC 60814:1997 Insulating liquids. Oilimpregnated paper and pressboard. Determination of water by automatic coulometric Karl Fischer titration, BSI, 1998. 